\title{
An in vitro and in vivo study of gemcitabine-loaded albumin nanoparticles in a pancreatic cancer cell line
}

This article was published in the following Dove Press journal:

International Journal of Nanomedicine

30 October 2015

Number of times this article has been viewed

Xinzhe Yu, ${ }^{1, *}$ Yang $D i,{ }^{2, *}$ Chao Xie, ${ }^{3}$ Yunlong Song, ${ }^{4}$ Hang $\mathrm{He},{ }^{2}$ Hengchao Li, Xinming $\mathrm{Pu},{ }^{5}$ Weiyue $\mathrm{Lu},{ }^{3}$ Deliang $\mathrm{Fu},{ }^{2}$ Chen Jin'

'Pancreatic Surgery Department, Huashan Hospital, ${ }^{2}$ Pancreatic Disease Institute, Fudan University, ${ }^{3}$ School of Pharmacy \& Key Laboratory of Smart Drug Delivery, Fudan University, ${ }^{4}$ School of Pharmacy, The Second Military Medical University, ${ }^{5}$ The State Key Laboratory of Molecular Engineering of Polymers, Department of Macromolecular Science, Fudan University, Shanghai, People's

Republic of China

*These authors contributed equally to this work

Video abstract

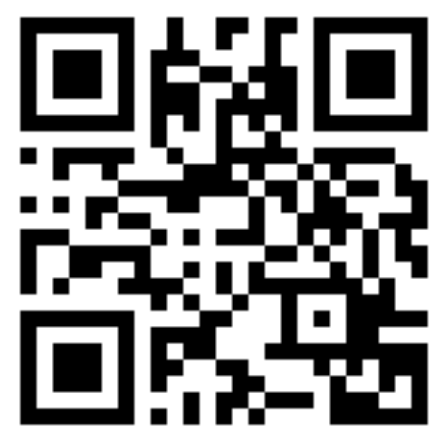

Point your SmartPhone at the code above. If you have a QR code reader the video abstract will appear. Or use: http://youtu.be/vPWpgACyn8w

Correspondence: Chen Jin

Pancreatic Surgery Department, Huashan Hospital, Fudan University, Shanghai

200040, People's Republic of China

Tel +86 I36 0I 98 I I74

Email galleyking@hotmail.com
Background and objectives: Gemcitabine (Gem) is far from satisfactory as the first-line regimen for pancreatic cancer, and the emergence of albumin nanoparticles offers new hope for the delivery of Gem. In this study, Gem-loaded human serum albumin nanoparticles (Gem-HSA-NPs) were successfully synthesized, characterized, and tested on a BxPC-3 cell line both in vitro and in vivo.

Materials and methods: $4-N$-myristoyl-gemcitabine (Gem-C14) was obtained first by coupling myristoyl with the 4-amino group of Gem. The Gem-HSA-NPs were then prepared by nanoparticle albumin-bound technology and characterized for particle size, zeta potential, morphology, encapsulation efficiency, drug-loading efficiency, and release characteristics. Using both in vitro and in vivo studies, Gem-C14 and Gem-HSA-NPs were tested on the human pancreatic cancer cell line BxPC-3.

Results: Gem-HSA-NPs showed an average particle size of $150 \pm 27 \mathrm{~nm}$, and with an encapsulation rate of $82.99 \% \pm 3.5 \%$ and a drug-loading rate of $10.42 \% \pm 3.5 \%$, they exhibited a favorable controlled- and sustained-release nature. In in vitro, Gem-C14 was equivalent in cytotoxicity to Gem. In in vivo, the Gem-HSA-NPs exhibited the strongest inhibitory effect on tumor growth but the lowest toxicity among the four groups.

Conclusion: The enhanced in vivo efficacy of Gem-HSA-NPs toward the pancreatic cancer cell line suggests their potential role for use in the clinical field.

Keywords: pancreatic cancer, gemcitabine, albumin nanoparticles, BxPC-3 cell line, in vitro study, in vivo study

\section{Introduction}

Pancreatic cancer is one of the most lethal diseases, making it a major health concern worldwide. According to the statistics, pancreatic cancer is the fourth, fifth, and seventh leading cause of cancer-related deaths in the USA, the European Union, and People's Republic of China, respectively. ${ }^{1-3}$ Although great progress has been made for other cancers, pancreatic cancer still has a poor prognosis, with a dismal 5-year survival rate $<5 \%{ }^{4}$ Surgery is the only effective cure, but only $20 \%$ of patients are eligible for this approach, and chemotherapy remains the standard treatment for most patients with pancreatic cancer. ${ }^{5}$

Currently, gemcitabine (Gem) (2',2'-difluoro-2'-deoxycytidine) is recommended as the first-line regimen for advanced pancreatic cancer. ${ }^{6}$ However, because of its low molecular weight and high solubility in water, the clinical benefits of Gem are compromised by its short plasma half-life and relative low concentration around tumor sites. ${ }^{7}$ Furthermore, in order to achieve optimal clinical efficacy, a schedule 
of frequent high doses is required, which in turn leads to significant side effects. ${ }^{8,9}$ Therefore, a search for novel therapeutic strategies is imperative. Albumin nanoparticles are increasingly being used as drug carriers for effective accumulation within tumor tissues ${ }^{10,11}$ via the following two mechanisms: passive targeting via the enhanced permeability and retention (EPR) effect ${ }^{12}$ and active targeting via two albumin-binding proteins, ${ }^{13,14}$ one is gp60 receptor, which is on the tumor endothelium, ${ }^{15,16}$ and the other is a secreted protein, acidic and rich in cysteine, an albumin "sticker" enriched in the tumor interstitium. ${ }^{17,18}$ Compared with artificial materials, albumin is nontoxic, biocompatible, biodegradable, and reproduced in the human body. ${ }^{19,20}$

Nanoparticle albumin-bound (nab)-paclitaxel is another emerging tool for combating late-stage pancreatic cancer. Encouraging results from a number of clinical trials have paved the way for nab-paclitaxel to be approved as the first-line option in advanced pancreatic cancer. ${ }^{21,22}$ Based on the nab technology, hydrophobic agents can be encapsulated into a hydrophilic exterior core, thereby becoming soluble nanoparticles. ${ }^{23}$ The nanoparticles are physically stable and have a reproducible, narrow range of diameters $(50-150 \mathrm{~nm})$ with a mean value of $130 \mathrm{~nm} \cdot{ }^{24}$ Their negatively charged albumin surfaces repel each other, allowing the particles to remain in homogenous suspension and avoiding flocculation. ${ }^{25}$ As described earlier, nab-paclitaxel also harnesses the two albumin-binding proteins to increase the targeting efficiency of the drug to the tumor tissues. Therefore, we wondered whether using nab technology we could encapsulate Gem into albumin nanoparticles and thus optimize the delivery of Gem into pancreatic cancer cells.

We have previously reported on the use of albumin nanoparticles as drug carriers to encapsulate Gem in pancreatic cancer, ${ }^{26-28}$ in which two sizes of Gem-loaded albumin nanoparticles (110 nm and $406 \mathrm{~nm}$ ) were successfully prepared. Both indicated improved antitumor cytotoxicity and prolonged drug release characteristics. However, using the desolvation-crosslinking method, these albumin nanoparticles showed poor stability, a lack of uniformity, and nonreproducibility, but with the advent of nab technology, these obstacles can be well addressed. To our knowledge, this article is the first report on the synthesis of 4-N-myristoyl-gemcitabine (Gem-C14) and also the first study report on using nab technology to encapsulate Gem-C14 into Gem-loaded human serum albumin nanoparticles (Gem-HSA-NPs). We also conducted in vitro and in vivo studies of Gem-HSA-NP in the pancreatic cancer cell line BxPC-3.

\section{Materials and methods Chemical and reagents}

Gemcitabine (crude drug) was obtained from Eli Lilly and Company (Indianapolis, IN, USA), and the purity was $>99.9 \%$. HSA $(20 \%, 50 \mathrm{~mL})$ was purchased from Baxter AG (Vienna, Austria). Normal saline (NS) was purchased from Shanghai Baxter Healthcare Co., Ltd. (Shanghai, People's Republic of China). Absolute ethanol and chloroform were purchased from Sinopharm Chemical Reagent Co., Ltd. (Shanghai, People's Republic of China); deionized water was purchased from Fudan University (Shanghai, People's Republic of China); and Roswell Park Memorial Institute (RPMI)-1640 culture medium and fetal bovine serum were obtained from Thermo Fisher Scientific (Waltham, MA, USA). Double stain Hoechst/PI kit and myristic acid (C14) were purchased from Sigma-Aldrich Co. (St Louis, MO, USA). Cell Counting Kit-8 (CCK-8) was purchased from Dojindo (Kyushu, Japan). Terminal deoxynucleotidyl transferase dUTP nick end labeling (TUNEL) kit was purchased from Hoffman-La Roche Ltd. (Basel, Switzerland). Phosphate-buffered saline (PBS) and other reagents were prepared in our laboratory. All the solvents and chemicals were of analytical grade.

\section{Synthesis of Gem-CI4}

Myristic acid was dissolved in tetrahydrofuran, and 1,1'carbonyldiimidazole was then added to the solution. The mixture was stirred at room temperature for 20 minutes to produce solution A. A solution of Gem base in anhydrous $\mathrm{CH}_{3} \mathrm{CN}$ was mixed with pyridine and trimethylsilyl chloride. The solution was stirred at room temperature for 20 minutes to produce solution B. Solution A was added dropwise to solution $\mathrm{B}$. The resulting mixture was stirred for 12 hours at $60^{\circ} \mathrm{C}$. Methanol and trifluoroacetic acid were added dropwise into the solution. The reaction mixture was stirred at room temperature for 10 minutes and then concentrated and dried under vacuum. The solid was dissolved in EtOAc (Ethyl acetate) and washed with brine, saturated $\mathrm{NaHCO}_{3}$ solution, and water. The organic layer was dried over $\mathrm{MgSO}_{4}$ and evaporated. The solid was purified by silica gel column flash chromatography and eluted with a mixture of methanol/chloroform (2:100) to produce the crude product, which was further recrystallized in methanol to generate the final product at a yield of $13 \%$.

\section{Preparation and characterization of Gem-HSA-NPs}

Gem-HSA-NPs were prepared by nab technology as previously reported..$^{29}$ Briefly, $12 \mathrm{~mL} \mathrm{20 \%} \mathrm{HSA} \mathrm{was} \mathrm{mixed}$ with $68 \mathrm{~mL}$ pure water. Separately, $100 \mathrm{mg}$ Gem-C14 was dissolved in $1 \mathrm{~mL}$ of chloroform saturated with pure water. 
These two solutions were then fully mixed and homogenized (Nano DeBEE; BEE International, South Easton, MA, USA) at 20,000 psi for nine cycles. The generated colloid was rotary evaporated to remove the chloroform at $25^{\circ} \mathrm{C}$ for 15 minutes under vacuum. The obtained nanoparticles were then filtered through a $0.25-\mu \mathrm{m}$ membrane syringe filter, and the solvent was removed by lyophilization for 48 hours at $-80^{\circ} \mathrm{C}$. The obtained Gem-HSA-NPs powder was vacuum dried for 48 hours and stored at room temperature.

The particle sizes and zeta potential were determined using Zetasizer (Malvern Instruments, Malvern, UK) at a scattering angle of $120^{\circ}$. The nanoparticle suspension was added dropwise onto the copper grids, which were then dried at room temperature. Transmission electron microscopy images of the nanoparticles were acquired (Hitachi H-600; Hitachi Ltd., Tokyo, Japan).

For drug-loading efficiency and encapsulation efficiency, preweighted amount of Gem-HSA-NPs was taken in distilled water and sonicated to extract Gem. The extracted Gem was analyzed by high-performance liquid chromatography-UV assay. Data were collected and calculated using the following formula:

$\begin{aligned} & \text { Encapsulation } \\ & \text { efficiency }(\%)\end{aligned}=\frac{\text { Amount of gemcitabine encapsulated }}{\text { Total gemcitabine added }} \times 100$

$\begin{aligned} & \text { Drug loading } \\ & \text { efficiency }(\%)\end{aligned}=\frac{\text { Amount of gemcitabine encapsulated }}{\text { Total weight of nanoparticles }} \times 100$.

Gemcitabine release was monitored by dispersing $2 \mathrm{mg}$ of Gem-HSA-NPs in 3 mL PBS (pH 7.4). The samples were kept in temperature-controlled horizontal shaker (Ningbo Biotech Scientific Instrument Co., Ltd., Ningbo, People's Republic of China), and samples were drawn at different time points and analyzed using ultraviolet-visible (UV-Vis) spectrophotometer.

\section{Mice and cell culture}

The BALB/c-nu/nu female mice (weighing $20 \pm 2 \mathrm{~g}$ ) were purchased from the Charles River Laboratories (Wilmington, MA, USA) and housed in a pathogen-free animal facility. The temperature was maintained at $24^{\circ} \mathrm{C}$ with a humidity of $50 \%-60 \%$, and the mice were subjected to a $10 / 14$-hour light/dark cycle. All animal studies were in compliance with the approved animal protocols and the guidelines of the Institutional Animal Care and Use Committee of Fudan University. The BxPC-3 (human pancreatic cancer cell line, originally from the American Type Culture Collection) was purchased from the Shanghai Branch of the Chinese Academy of Sciences (Shanghai, People's Republic of China). No ethics statement was required from the institutional review board for the use of these cell lines.

Cells were cultured at $37^{\circ} \mathrm{C}$ in the presence of $5 \% \mathrm{CO}_{2}$ and $95 \%$ air with $>95 \%$ humidity. Growth medium was RPMI-1640, containing 10\% fetal bovine serum, $100 \mathrm{U} / \mathrm{mL}$ penicillin, and $100 \mathrm{mg} / \mathrm{mL}$ streptomycin.

\section{Cell viability}

The in vitro cytotoxic effects of Gem, Gem-C14, and GemHSA-NPs on BxPC-3 cells were determined using the CCK-8 assay. Briefly, cells were seeded into 96-well culture plates $(10,000$ cells/well $)$ and incubated overnight at $37^{\circ} \mathrm{C}$, $5 \% \mathrm{CO}_{2}$. Cells were then incubated with various concentrations of Gem, Gem-C14, Gem-HSA-NPs, or sterile PBS for 48 hours. The cell viability was quantitated by reading the dye absorption at $450 \mathrm{~nm}$ with an automatic multiwall spectrophotometer. The cell inhibition rate $(\%)$ was calculated as follows: (1- [absorbance of the study group/absorbance of the control group]) $\times 100$.

\section{Apoptotic cell damages assays}

The apoptosis rate was assessed using the annexin V-fluorescein isothiocyanate (FITC)/PI according to the instructions of manufacturer. The BxPC-3 cells were grouped and treated as described for the CCK-8 assay. Briefly, BxPC-3 cells were placed in a six-well culture plate $\left(5 \times 10^{5}\right.$ cells/well). The cells in each group were collected through centrifugation at 1,000 rpm for $5 \mathrm{~min}$ utes, followed by washing with PBS for three times and resuspension in binding buffer. Then, $5 \mu \mathrm{L}$ of annexin V-FITC solution and $10 \mu \mathrm{L}$ of PI solution were added in turn. The stained cells were incubated for 15 minutes at room temperature in the dark. Finally, the suspension was subjected to the flow cytometry analysis (BD Biosciences, San Jose, CA, USA). All the experiments were performed in triplicate.

\section{In vivo antitumor study}

An animal model was obtained by subcutaneous injection of $1 \times 10^{7}$ BxPC-3 cells suspended in $100 \mu \mathrm{L}$ RPMI-1640 medium into the right flank of nude mice. ${ }^{30}$ Studies were done 2 weeks after implantation, when the tumors had reached $5.0 \mathrm{~mm}$ in diameter. The mice were then randomized into four groups ( $n=6$ per group) and treated with $100 \mu \mathrm{L}$ of Gem, Gem-C14, Gem-HSA-NPs, and NS through tail vein injection on days $0,3,6,9$, and 12 (Gem or equivalent dosage: $50 \mathrm{mg} / \mathrm{kg}$ body weight). Mice body weights and 
tumor sizes were monitored every 2 days until day 14 . Tumor volume was estimated using the formula: volume $\left(\mathrm{mm}^{3}\right)=($ length in $\mathrm{mm}) \times(\text { width in } \mathrm{mm})^{2} / 2$.

\section{TUNEL assays}

On day 14 , the mice were sacrificed by cervical dislocation, and the tumor mass was harvested, photographed, and then fixed with paraformaldehyde for 48 hours and embedded in paraffin. Each section was cut into $5 \mu \mathrm{m}$ slice and used for TUNEL staining according to the manufacturer's instructions. Nucleus staining was performed with 4',6-diamidino-2phenylindole (blue) to locate the nucleus, and the percentage of apoptosis-positive (red-stained) cells were visualized under a confocal microscope (Leica DMI 4000B; Leica Microsystems, Wetzlar, Germany).

\section{Statistical analysis}

All the data are presented as the mean \pm standard deviation. Significant differences between two groups were determined using Student's $t$-test, while multiple groups were analyzed by one-way analysis of variance with Fisher's least significant difference. All the statistical analyses were performed with SPSS software (Version 22.0; IBM Corporation, Armonk, NY, USA). A value of $P<0.05$ was considered significant.
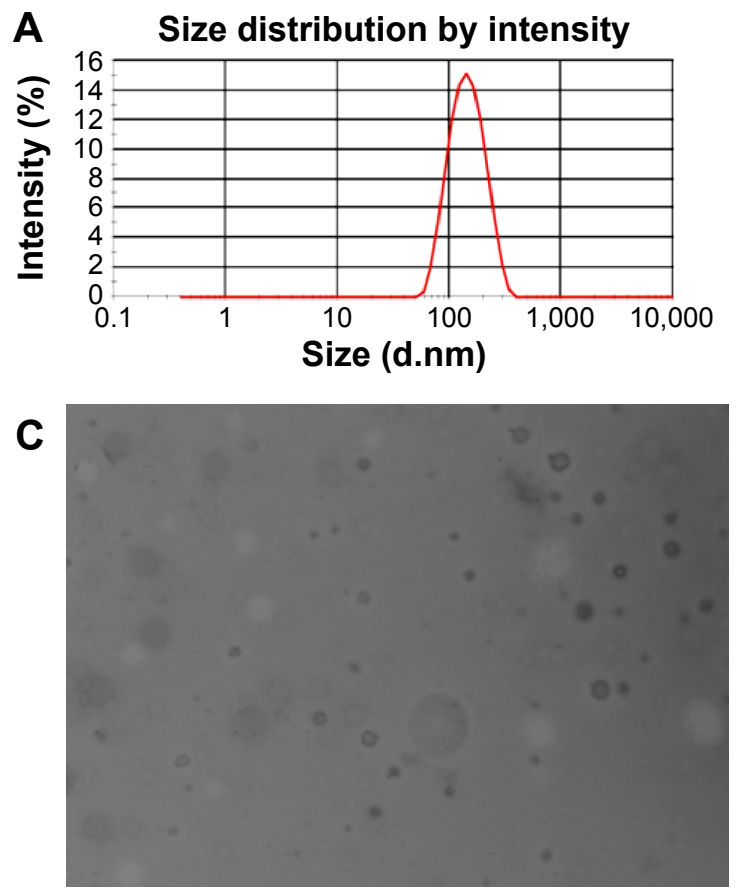

Original magnification $\times \mathbf{2 0 , 0 0 0}$

\section{Results}

\section{Characterization of the Gem-HSA-NPs}

The Gem-HSA-NPs were prepared using nab technology, by which Gem-C14 was mixed with HSA in an aqueous solvent and passed under high pressure through a jet to form drug albumin nanoparticles. As shown in Figure 1A, the average size of the nanoparticles was $150 \pm 27 \mathrm{~nm}$, and the polydispersity was $0.162 \pm 0.02$. Overall, the nanoparticles showed a narrow size distribution, suggesting their potential role as passively targeted antitumor agent. The nanoparticles showed a negative surface potential of $-10.2 \pm 3.2 \mathrm{mV}$ (Figure 1B), indicating their potential for prolonged blood circulation; nanoparticles with a positive surface charge would interact with serum protein in human blood. The morphology of the Gem-HSA-NPs was observed by transmission electron microscopy. As shown in Figure $1 C$, under $\times 20,000$ magnification, the Gem-HSA-NPs exhibited a nearly spherical shape with a moderate uniform particle size and even distribution. Under $\times 50,000$ magnification (Figure 1D), the nanoparticles had a brighter core surrounded by a dark membrane, which confirmed the distinct layer.

The encapsulation efficiency of Gem-HSA-NPs was $82.99 \% \pm 3.5 \%$, and the drug-loading efficiency was $10.42 \% \pm 3.5 \%$. The in vitro release profiles of Gem-HSA-NP were recorded in a cumulative release curve $>5$ days. As shown in Figure 2, for the 1st hour, $<20 \%$ of the drug was
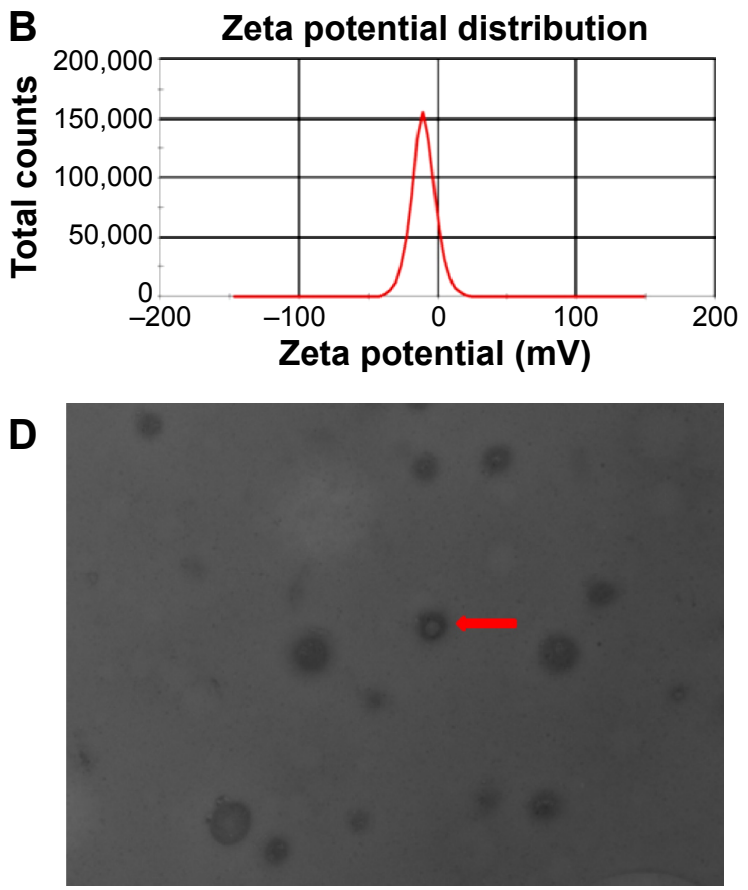

Original magnification $\times 50,000$

Figure I Particle size and size distribution of Gem-HSA-NP determined using a Malvern Zetasizer (A). Zeta potential spectrum of Gem-HSA-NP in PBS solution (B). TEM image of Gem-HSA-NP under $\times 20,000$ magnification (C). TEM image of Gem-HSA-NP under $\times 50,000$ magnification. The nanoparticles had a brighter core surrounded by a dark membrane (arrow), which confirmed its distinct layers (D).

Abbreviations: Gem-HSA-NP, gemcitabine-loaded human serum albumin nanoparticle; PBS, phosphate-buffered saline; TEM, transmission electron microscopy. 


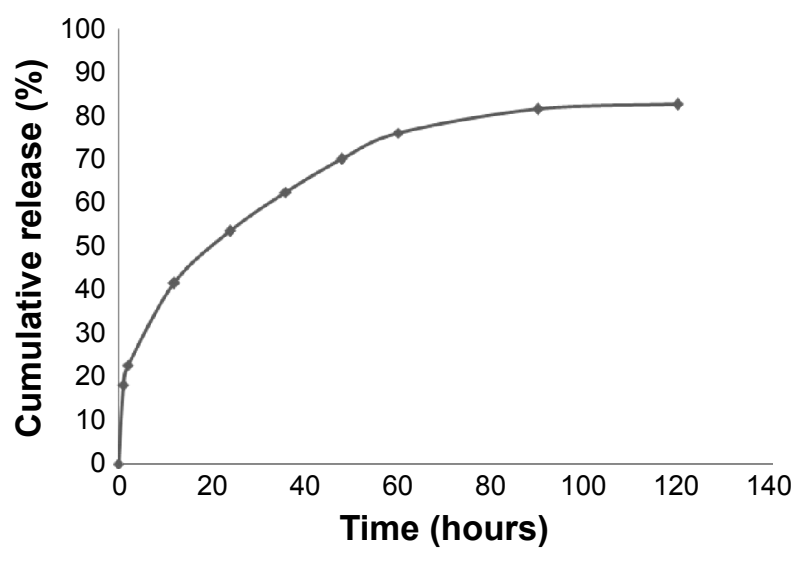

Figure 2 The cumulative release curve of Gem-HSA-NPs.

Notes: For the Ist hour, $<20 \%$ of drugs were released and thereafter, the nanoparticles showed an accelerated release, whereas over half of Gem were released at the time of the 24 th hour. For the 48 th hour, $>70 \%$ of Gem were released, followed by a slow sustained-release character. Until the 90 th hour, a relative release peak of $81.5 \%$ was observed, and this remained stable for the next 30 hours.

Abbreviations: Gem-HSA-NP, gemcitabine-loaded human serum albumin nanoparticle; Gem, gemcitabine.

released, which shows that Gem-HSA-NPs can retain their stability in solution. Thereafter, the nanoparticles showed an accelerated release, where more than half of the Gem was released by 24 hours. By 48 hours, $>70 \%$ of the Gem had been released; this was followed by a slow, sustained release. By hour 90, a relative release peak of $81.5 \%$ was observed, and this remained stable for the next 30 hours.

\section{In vitro results}

The in vitro cytotoxicity of Gem-HSA-NP was evaluated on the human pancreatic cancer cell line BxPC-3 via the CCK-8 assay. As shown in Figure 3, the 1-8 surveying points stood for the respective concentrations of $0.01 \mu \mathrm{g} / \mathrm{mL}, 0.04 \mu \mathrm{g} / \mathrm{mL}$, $0.2 \mu \mathrm{g} / \mathrm{mL}, 0.5 \mu \mathrm{g} / \mathrm{mL}, 1 \mu \mathrm{g} / \mathrm{mL}, 5 \mu \mathrm{g} / \mathrm{mL}, 10 \mu \mathrm{g} / \mathrm{mL}$, and $50 \mu \mathrm{g} / \mathrm{mL}$. All three drugs inhibited cell growth in a dosedependent manner, in which Gem exhibited the highest cytotoxicity, followed by Gem-C14 and then Gem-HSA-NPs. The $\mathrm{IC}_{50}$ of Gem is $\sim 0.2 \mu \mathrm{g} / \mathrm{mL}$, that of Gem-C14 is $0.35 \mu \mathrm{g} / \mathrm{mL}$, and that of Gem-HSA-NPs is $0.4 \mu \mathrm{g} / \mathrm{mL}$.

We also assessed the apoptosis assays of the three groups. As shown in Figure 4, apoptosis and necrosis can be found in all treatment groups, and the total apoptosis rates (including early and late-stage apoptosis) of the three groups (Gem, Gem-C14, and Gem-HSA-NPs) were 34.94\% $2.5 \%$, $37.72 \% \pm 1.8 \%$, and $37.48 \% \pm 2.6 \%$, respectively, which were not statistically significant $(P>0.05)$.

\section{In vivo results}

To evaluate the antitumor activity of Gem-HSA-NPs in a human pancreatic cancer xenograft model in vivo, we examined tumor growth and body weight changes in nude

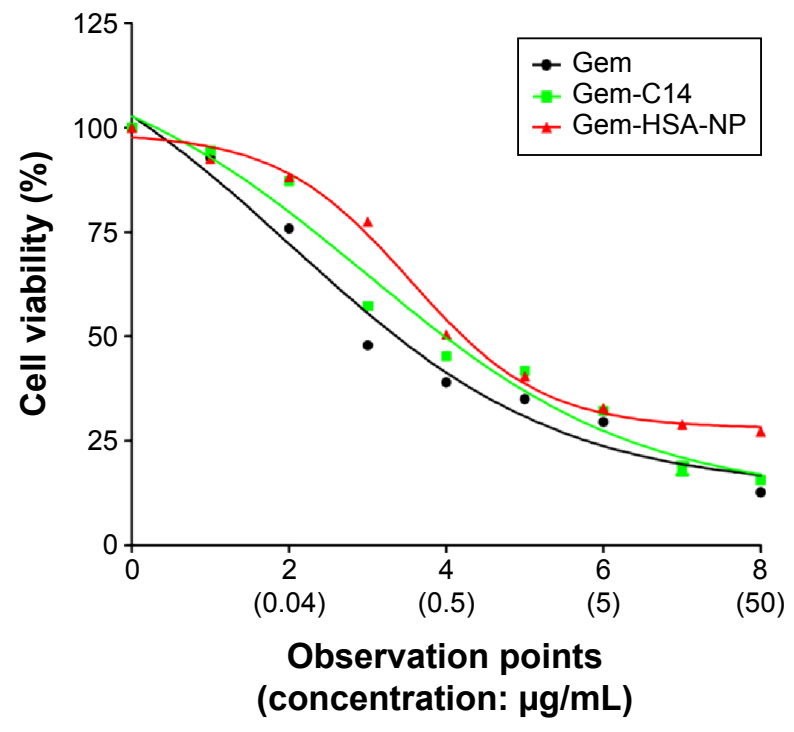

Figure 3 In vitro cytotoxicity assay of Gem-HSA-NP on BxPC-3 cell line.

Notes: The I-8 surveying points stood for $0.01 \mu \mathrm{g} / \mathrm{mL}, 0.04 \mu \mathrm{g} / \mathrm{mL}, 0.2 \mu \mathrm{g} / \mathrm{mL}$, $0.5 \mu \mathrm{g} / \mathrm{mL}, 1 \mu \mathrm{g} / \mathrm{mL}, 5 \mu \mathrm{g} / \mathrm{mL}, 10 \mu \mathrm{g} / \mathrm{mL}$, and $50 \mu \mathrm{g} / \mathrm{mL}$, respectively. All three drugs inhibited cell growth in a dose-dependent manner, in which Gem exhibited the highest cytotoxicity, followed by Gem-Cl4, whereas the Gem-HSA-NP showed the lowest cytotoxicity level. The $\mathrm{IC}_{50}$ of $\mathrm{Gem}$ is $\sim 0.2 \mu \mathrm{g} / \mathrm{mL}$, that of $\mathrm{Gem}-\mathrm{Cl} 4$ is $0.35 \mu \mathrm{g} / \mathrm{mL}$, and that of Gem-HSA-NPs is $0.4 \mu \mathrm{g} / \mathrm{mL}$.

Abbreviations: Gem-HSA-NP, gemcitabine-loaded human serum albumin nanoparticle; Gem, gemcitabine; Gem-Cl4, 4-N-myristoyl-gemcitabine.

mice treated with Gem, Gem-C14, Gem-HSA-NPs, and NS. During the entire study, no mice died from any treatmentrelated cause. As shown in Figure $5 \mathrm{~A}$ and $\mathrm{B}, \sim 2$ weeks after the treatment, the tumor volume of the mice receiving the Gem formulation followed the order: Gem-HSA-NPs $<$ Gem or Gem-C14 < NS. Tumors in mice treated with the GemHSA-NPs grew significantly slower than those in the other groups. Figure 5C shows that the average tumor volumes of mice treated with Gem-HSA-NPs were half the size of those in the Gem group and one-sixth the size of the NS group $(P<0.05)$, whereas, in the Gem and Gem-C14 groups, the tumor volumes were almost the same $(P>0.05)$.

In order to evaluate the safety of the Gem-HSA-NPs, mouse body weights were monitored as a marker of overall toxicity. As shown in Figure 5D, the weight profiles showed a sudden loss on day 4 in both the Gem and Gem-C14 groups, whereas, in the other two groups, the weight remained stable. On day 14, mice in the Gem and Gem-C14 groups suffered a $10 \%$ body weight loss $(P<0.05)$ compared with those in the Gem-HSA-NPs group, which indicates rapid disease progression and more severe toxicity.

As shown in Figure 6, the TUNEL staining was used to label apoptotic cells, indicated by red fluorescence in the tumor section (with blue nuclei). The degree of apoptosis varied as follows: Gem-HSA-NPs $>$ Gem-C14 $>$ Gem $>$ normal control, indicating that the Gem-HSA-NPs were the most effective in inducing apoptosis of tumor cells (see Figure S1 for the data in detail). 


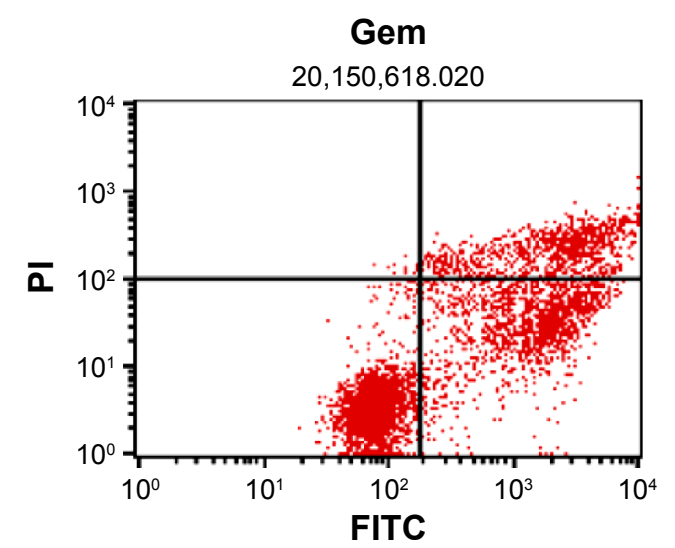

Gem-HSA-NP

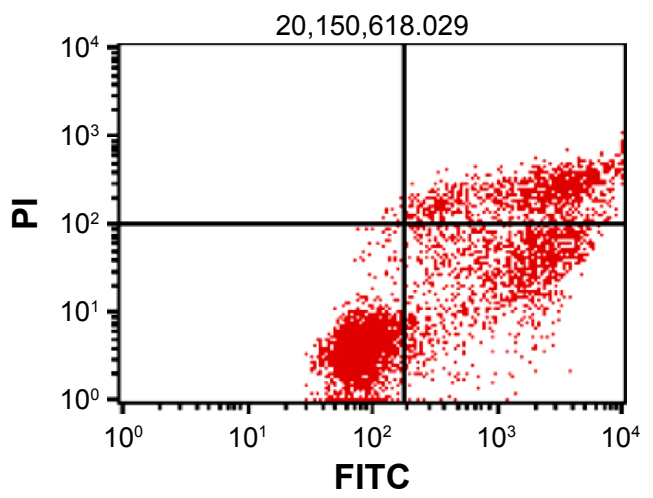

Gem-C14

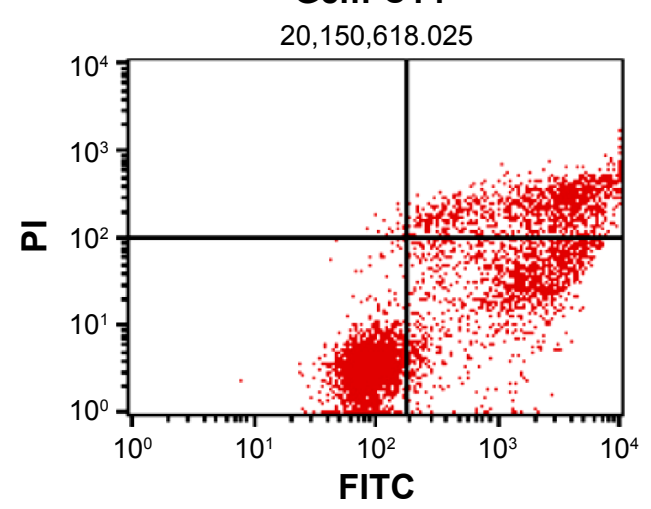

Figure 4 Annexin V-FITC/PI graphs of flow cytometry for Gem, Gem-CI4, and Gem-HSA-NP.

Notes: The apoptotic rates of the three drugs were $34.94 \% \pm 2.5 \%, 37.72 \% \pm 1.8 \%$, and $37.48 \% \pm 2.6 \%$, respectively. Numbers in the quadrants are percentage of cells in early apoptosis (UR), late apoptosis or necrosis (LR), and cell debris (UL).

Abbreviations: FITC, fluorescein isothiocyanate; Gem, gemcitabine; Gem-HSA-NP, gemcitabine-loaded human serum albumin nanoparticle; UR, upper right; LR, lower right; UL, upper left; PI, propidium iodide.

It should be noted here, however, that the distribution of red fluorescence may also represent blood vessels; therefore, we used 4',6-diamidino-2-phenylindole to mark the nuclei so as to differentiate the vessels from tumor cells.

\section{Discussion}

Being a nucleoside analog, Gem is rapidly converted into its inactive metabolite $\mathrm{dFdU}\left(2^{\prime}, 2^{\prime}\right.$-difluorodeoxyuridine) by cytidine deaminase after systemic administration. ${ }^{31}$ In addition, Gem is too hydrophilic to cross the cell membrane, which must rely on specialized nucleoside transporters, such as human equilibrative nucleoside transporter 1 and human equilibrative nucleoside transporter $2 .^{32}$ Several strategies have been proposed to overcome these deficiencies: ${ }^{7,33,34} 1$ ) to encapsulate Gem into nanoparticles with the advantages of an EPR effect and controlled-release characteristics; these materials can be albumin, liposome, polymeric compounds, etc and 2) to conjugate an acyl chain with the N-terminus of Gem, thereby enhancing its hydrophobicity. In this paper, we report the successful synthesis of Gem-loaded albumin nanoparticles based on the nab technology for the first time. The whole process can be divided into two steps: first, a lipophilic derivative of Gem, named Gem-C14, is obtained by coupling myristoyl with the 4-amino group of Gem and, second, under optimum circumstances, the Gem-HSA-NPs are synthesized using nab technology.

In recent years, a number of attempts have been made to encapsulate drugs into albumin nanoparticles based on the desolvation-crosslinking method. ${ }^{35-37}$ Previously, our research group also used a modified desolvation-crosslinking method to produce Gem-loaded albumin nanoparticles in two sizes (110 nm and $406 \mathrm{~nm})$. Although both these nanoparticles showed significant inhibitory effects on human pancreatic cancer cell lines, the inhibition rate was size dependent, and we found that $406 \mathrm{~nm}$ nanoparticles demonstrated greater antitumor activity. Particle size and size distribution have important effects on the stability of a nanoparticle system. It is generally believed that particles up to 100-200 nm can be internalized by receptor-mediated endocytosis, whereas larger particles have to be taken up by 
A

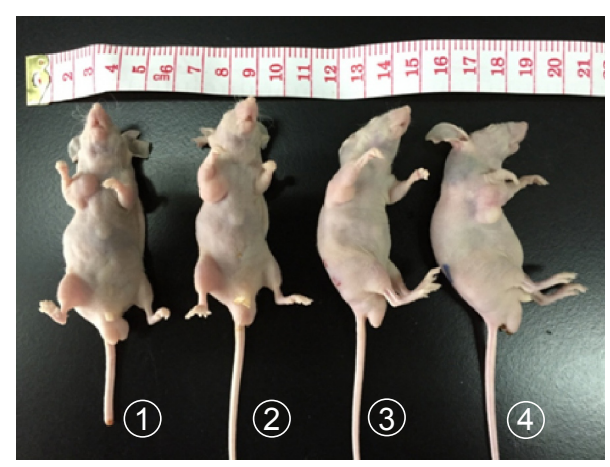

C

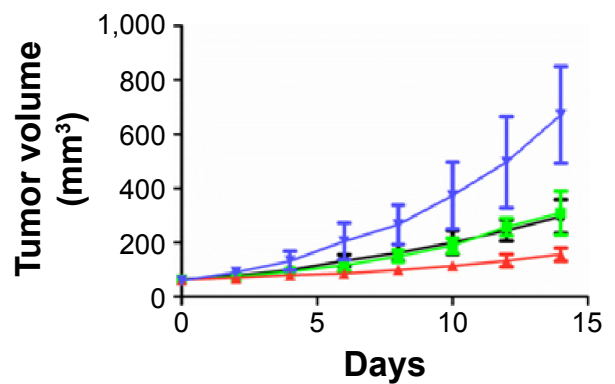

B

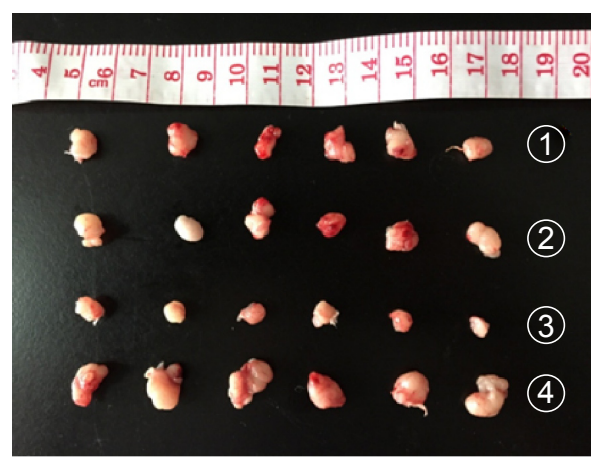

D

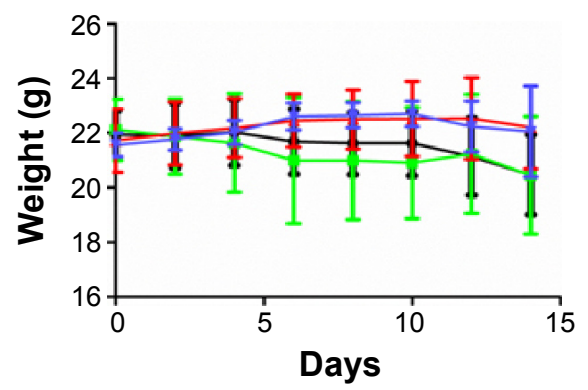

$\leftarrow$ Gem $=$ Gem-C14 $\neq$ Gem-HSA-NP $*$ NS

Figure 5 Representative photos of tumors on mice (A). Photographs of tumors harvested from each treatment group after various treatments (B). The tumor volume profiles of mice treated with Gem, Gem-Cl4, Gem-HSA-NP, and NS (C). The body weight profiles of mice treated with Gem, Gem-Cl4, Gem-HSA-NP, and NS (D). Notes: I-4 represent Gem, Gem-Cl4, Gem-HSA-NP, and NS group, respectively.

Abbreviations: Gem, gemcitabine; Gem-Cl4, 4-N-myristoyl-gemcitabine; Gem-HSA-NP, gemcitabine-loaded human serum albumin nanoparticle; NS, normal saline.
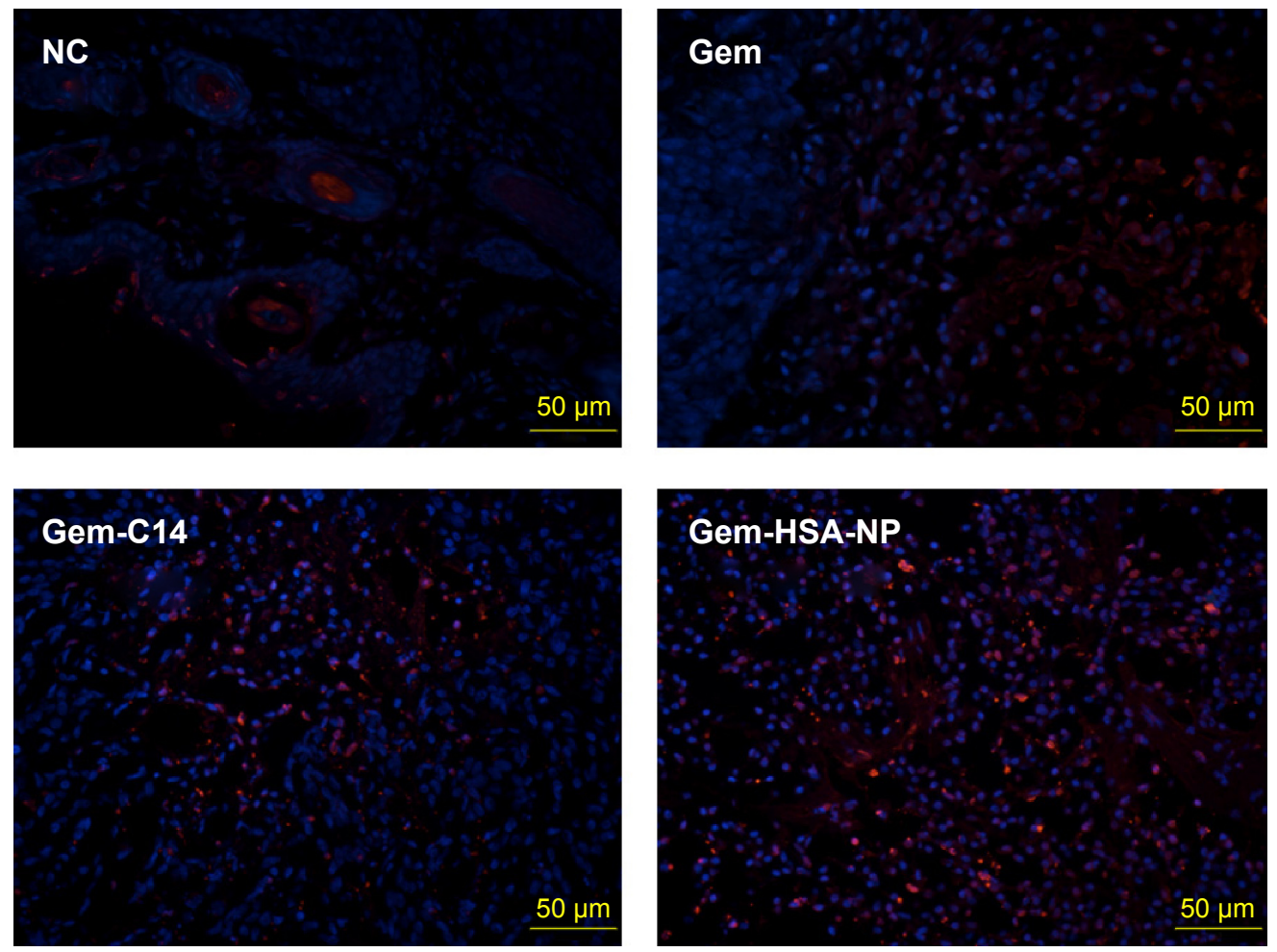

Figure 6 The merged TUNEL stainings showed tumor penetration of drugs on mice bearing subcutaneous pancreatic tumors on day I4 after IV administration.

Notes: Frozen sections were examined under a confocal microscope. Nuclei were stained with DAPI (blue), while red represented apoptotic cells. The bar indicated $50 \mu \mathrm{m}$. Abbreviations: TUNEL, transferase dUTP nick end labeling; IV, intravenous; DAPI, 4',6-diamidino-2-phenylindole; NC, negative control; Gem, gemcitabine; Gem-CI4, 4-Nmyristoyl-gemcitabine; Gem-HSA-NP, gemcitabine-loaded human serum albumin nanoparticle. 
phagocytosis. ${ }^{38,39}$ Nanoparticles are prone to aggregate and precipitate if the particles are large or the size distribution is not even. However, owing to the limitations of the desolvation-crosslinking method, it is difficult to control the size and size distribution of particles. With the emergence of nab-paclitaxel as the first-line therapy for advanced pancreatic cancer, more attention has been drawn to the new technology of albumin nanoparticle synthesis. This was developed by American BioScience Inc. and named nab technology, and is ideal for encapsulating lipophilic drugs into nanoparticles. Using this technology, the size of nanoparticles can be controlled in the range of 100-200 $\mathrm{nm}$. In addition, the nanoparticles are stable and evenly distributed. Besides nab-paclitaxel, Kim et $\mathrm{al}^{29}$ used nab technology to prepare curcumin-loaded albumin nanoparticles with a size range of 130-150 nm for intravenous administration. Similarly, in our study, the particle sizes of Gem-HSA-NPs were well controlled in the range of $130-160 \mathrm{~nm}$, with an average of $150 \pm 27 \mathrm{~nm}$. During antitumor therapy, this specific size can maximize the EPR effect while avoiding being taken up by the macrophages as well as the reticuloendothelial system.

Another concern may be the potential immunologic response caused by bovine serum albumin, so we used human serum albumin to encapsulate the Gem, as Gem is water soluble and must therefore be transformed into a liposoluble state in order to be encapsulated by nab technology. In our study, we increased the lipophilicity of Gem by coupling the 4-amino group with C14 linear-chain acyl derivatives. Another concern may be raised in terms of the therapeutic efficacy after derivatization of Gem. Both our in vitro and in vivo studies confirmed that Gem-C14 showed equal or even superior efficacy to Gem, and several other reports also support this conclusion., ${ }^{2,40,41}$ This enhanced antitumor activity may be attributed to the fact that, on the one hand, Gem-C14 has the ability to cross epithelial cells and localize in tumors independent of human equilibrative nucleoside transporter 1, and on the other hand, the metabolic enzyme intercellular carboxylesterase 2 is key for transforming Gem-C14 into Gem, which hydrolyzes the prodrug and confers prodrug sensitivity to cancer cells. ${ }^{42}$ Theoretically, the albumin nanoparticles have advantages over others in being nontoxic, biocompatible, and biodegradable..$^{43}$ Besides the EPR effect, the interplay of two albumin-binding proteins may facilitate the uptake and retention of albumin nanoparticles in tumor tissues. ${ }^{44}$ Our results are also in accordance with this hypothesis. Our in vivo study showed that after five cycles of drug administration, the mice in the Gem and Gem-C14 groups lost $10 \%$ of body weight, whereas in the NS and Gem-HSA-NPs groups, the average body weights were almost unchanged. With regard to inhibition of tumor growth, the Gem-HSA-NPs exhibited the strongest inhibitory effect. Furthermore, TUNEL staining also indicated that the Gem-HSA-NPs were the most effective in inducing apoptosis of tumor cells, which were mainly distributed evenly both intratumorally and around tumor vessels. As described earlier, a combined effect of EPR and the active targeting approach of albumin nanoparticles could be the main reasons for this significant suppression of tumor growth.

Compared with the in vivo study, the in vitro results showed no significant differences among the Gem, Gem-C14, and Gem-HSA-NPs groups. There may be two reasons for this: first, with Gem-HSA-NPs, there can be no effect of EPR in vitro. Second, the Gem-HSA-NPs revealed a controlledrelease nature: by hour 120 , only $82.6 \%$ of Gem had been released from the nanoparticles. However, the observation period for both in vitro studies was only 48 hours.

Last but not least, the strength of this study is obvious. This is the first report on the synthesis of Gem-C14, which demonstrates an equal or superior therapeutic effect to Gem both in vitro and in vivo. Furthermore, we were the first to use nab technology to encapsulate Gem-C14 into Gem-HSA-NPs and thereby showed a significantly improved antitumor efficacy over both Gem and Gem-C14. Finally, the limitation of this study is the subcutaneous tumor model, which cannot mimic the real tumor microenvironment of the human body (hypoxia and full of stroma). Although the in vivo results so far are exciting, the delivery of drugs to pancreatic cancer cells is still a challenge in the clinic. Another drawback of current Gem-HSA-NP is its lack of tumor-targeting ability; we are now preparing to attach cyclic arginine-glycine-aspartic-conjugated peptides to it, thereby strengthening its tumor-targeting ability.

\section{Conclusion}

In summary, we have successfully synthesized Gem-HSANPs. Our in vivo results confirm that these prepared GemHSA-NPs can more efficiently inhibit tumor growth in a pancreatic cancer cell line while showing moderate toxicity. However, further studies must be performed to optimize this therapy for clinical use.

\section{Acknowledgment}

This work was financially supported by the National Natural Science Foundation of China (81201896 and 81071884) and the Research Fund for the Doctoral Program of Higher Education of China (20130071110052 and 20110071110065).

\section{Disclosure}

The authors report no conflicts of interest in this work. 


\section{References}

1. Siegel R, Ma J, Zou Z, Jemal A. Cancer statistics, 2014. CA Cancer J Clin. 2014;64(1):9-29.

2. Bildstein L, Dubernet C, Marsaud V, et al. Transmembrane diffusion of gemcitabine by a nanoparticulate squalenoyl prodrug: an original drug delivery pathway. J Control Release. 2010;147(2):163-170.

3. Guo L, Fan L, Pang Z, et al. TRAIL and doxorubicin combination enhances anti-glioblastoma effect based on passive tumor targeting of liposomes. J Control Release. 2011;154(1):93-102.

4. Peixoto RD, Ho M, Renouf DJ, et al. Eligibility of metastatic pancreatic cancer patients for first-line palliative intent nab-paclitaxel plus gemcitabine versus FOLFIRINOX. Am J Clin Oncol. Epub 2015 Apr 1.

5. Conroy T, Desseigne F, Ychou M, et al; Groupe Tumeurs Digestives of Unicancer; PRODIGE Intergroup. FOLFIRINOX versus gemcitabine for metastatic pancreatic cancer. $N$ Engl J Med. 2011;364(19): $1817-1825$.

6. King RS. Gemcitabine. New first-line therapy for pancreatic cancer. Cancer Pract. 1996;4(6):353-354.

7. Moysan E, Bastiat G, Benoit JP. Gemcitabine versus modified gemcitabine: a review of several promising chemical modifications. Mol Pharm. 2013;10(2):430-444.

8. Nakano Y, Tanno S, Koizumi K, et al. Gemcitabine chemoresistance and molecular markers associated with gemcitabine transport and metabolism in human pancreatic cancer cells. Br J Cancer. 2007;96(3): 457-463.

9. Andersson R, Aho U, Nilsson BI, et al. Gemcitabine chemoresistance in pancreatic cancer: molecular mechanisms and potential solutions. Scand J Gastroenterol. 2009;44(7):782-786.

10. Sheng Z, Hu D, Zheng M, et al. Smart human serum albuminindocyanine green nanoparticles generated by programmed assembly for dual-modal imaging-guided cancer synergistic phototherapy. ACS Nano. 2014;8(12):12310-12322.

11. Kratz F. A clinical update of using albumin as a drug vehicle - a commentary. J Control Release. 2014;190:331-336.

12. Maeda H, Wu J, Sawa T, Matsumura Y, Hori K. Tumor vascular permeability and the EPR effect in macromolecular therapeutics: a review. J Control Release. 2000;65(1-2):271-284.

13. Gradishar WJ. Albumin-bound paclitaxel: a next-generation taxane Expert Opin Pharmacother. 2006;7(8):1041-1053.

14. Schnitzer JE, Oh P. Albondin-mediated capillary permeability to albumin. Differential role of receptors in endothelial transcytosis and endocytosis of native and modified albumins. J Biol Chem. 1994;269(8): 6072-6082.

15. Tiruppathi C, Song W, Bergenfeldt M, Sass P, Malik AB. Gp60 activation mediates albumin transcytosis in endothelial cells by tyrosine kinase-dependent pathway. J Biol Chem. 1997;272(41): 25968-25975

16. John TA, Vogel SM, Minshall RD, Ridge K, Tiruppathi C, Malik AB. Evidence for the role of alveolar epithelial gp60 in active transalveolar albumin transport in the rat lung. J Physiol. 2001;533(pt 2): 547-559.

17. Neuzillet C, Tijeras-Raballand A, Cros J, Faivre S, Hammel P, Raymond E. Stromal expression of SPARC in pancreatic adenocarcinoma. Cancer Metastasis Rev. 2013;32(3-4):585-602.

18. Hoang B, Ernsting MJ, Roy A, Murakami M, Undzys E, Li SD. Docetaxelcarboxymethylcellulose nanoparticles target cells via a SPARC and albumin dependent mechanism. Biomaterials. 2015;59:66-76.

19. Kratz F. Albumin as a drug carrier: design of prodrugs, drug conjugates and nanoparticles. J Control Release. 2008;132(3):171-183.

20. Elzoghby AO, Samy WM, Elgindy NA. Albumin-based nanoparticles as potential controlled release drug delivery systems. J Control Release. 2012;157(2):168-182.

21. Von Hoff DD, Ervin T, Arena FP, et al. Increased survival in pancreatic cancer with nab-paclitaxel plus gemcitabine. NEnglJMed.2013;369(18): 1691-1703.

22. Von Hoff DD, Ramanathan RK, Borad MJ, et al. Gemcitabine plus nab-paclitaxel is an active regimen in patients with advanced pancreatic cancer: a phase I/II trial. J Clin Oncol. 2011;29(34):4548-4554.
23. Li C, Li Y, Gao Y, et al. Direct comparison of two albumin-based paclitaxel-loaded nanoparticle formulations: is the crosslinked version more advantageous? Int J Pharm. 2014;468(1-2):15-25.

24. Montero AJ, Adams B, Diaz-Montero CM, Gluck S. Nab-paclitaxel in the treatment of metastatic breast cancer: a comprehensive review. Expert Rev Clin Pharmacol. 2011;4(3):329-334.

25. Yardley DA. nab-Paclitaxel mechanisms of action and delivery. J Control Release. 2013;170(3):365-372.

26. Li JM, Chen W, Wang H, et al. Preparation of albumin nanospheres loaded with gemcitabine and their cytotoxicity against BXPC-3 cells in vitro. Acta Pharmacol Sin. 2009;30(9):1337-1343.

27. Ji S, Xu J, Zhang B, et al. RGD-conjugated albumin nanoparticles as a novel delivery vehicle in pancreatic cancer therapy. Cancer Biol Ther. 2012;13(4):10.

28. Li J, Di Y, Jin C, et al. Gemcitabine-loaded albumin nanospheres (GEM-ANPs) inhibit PANC-1 cells in vitro and in vivo. Nanoscale Res Lett. 2013;8(1):176.

29. Kim TH, Jiang HH, Youn YS, et al. Preparation and characterization of water-soluble albumin-bound curcumin nanoparticles with improved antitumor activity. Int J Pharm. 2011;403(1-2):285-291.

30. Sandoval MA, Sloat BR, Lansakara-P DS, et al. EGFR-targeted stearoyl gemcitabine nanoparticles show enhanced anti-tumor activity. J Control Release. 2012;157(2):287-296.

31. Paolino D, Cosco D, Racanicchi L, et al. Gemcitabine-loaded PEGylated unilamellar liposomes vs GEMZAR: biodistribution, pharmacokinetic features and in vivo antitumor activity. J Control Release. 2010;144(2): 144-150.

32. Tao XM, Wang JC, Wang JB, et al. Enhanced anticancer activity of gemcitabine coupling with conjugated linoleic acid against human breast cancer in vitro and in vivo. Eur J Pharm Biopharm. 2012;82(2):401-409.

33. Federico C, Morittu VM, Britti D, Trapasso E, Cosco D. Gemcitabineloaded liposomes: rationale, potentialities and future perspectives. Int J Nanomedicine. 2012;7:5423-5436.

34. Wang L, An Y, Yuan C, et al. GEM-loaded magnetic albumin nanospheres modified with cetuximab for simultaneous targeting, magnetic resonance imaging, and double-targeted thermochemotherapy of pancreatic cancer cells. Int J Nanomedicine. 2015;10:2507-2519.

35. Chitkara D, Kumar N. BSA-PLGA-based core-shell nanoparticles as carrier system for water-soluble drugs. Pharm Res. 2013;30(9): 2396-2409.

36. Yang R, An Y, Miao F, Li M, Liu P, Tang Q. Preparation of folic acid-conjugated, doxorubicin-loaded, magnetic bovine serum albumin nanospheres and their antitumor effects in vitro and in vivo. Int $J$ Nanomedicine. 2014;9:4231-4243.

37. Dubey RD, Alam N, Saneja A, et al. Development and evaluation of folate functionalized albumin nanoparticles for targeted delivery of gemcitabine. Int J Pharm. 2015;492(1-2):80-91.

38. Yin Win K, Feng S-S. Effects of particle size and surface coating on cellular uptake of polymeric nanoparticles for oral delivery of anticancer drugs. Biomaterials. 2005;26(15):2713-2722.

39. Sykes EA, Chen J, Zheng G, Chan WC. Investigating the impact of nanoparticle size on active and passive tumor targeting efficiency. ACS Nano. 2014;8(6):5696-5706.

40. Stella B, Arpicco S, Rocco F, et al. Encapsulation of gemcitabine lipophilic derivatives into polycyanoacrylate nanospheres and nanocapsules. Int J Pharm. 2007;344(1-2):71-77.

41. Fang Y, Du F, Xu Y, et al. Enhanced cellular uptake and intracellular drug controlled release of VESylated gemcitabine prodrug nanocapsules. Colloids Surf B Biointerfaces. 2015;128:357-362.

42. Pratt SE, Durland-Busbice S, Shepard RL, Heinz-Taheny K, Iversen PW, Dantzig AH. Human carboxylesterase-2 hydrolyzes the prodrug of gemcitabine (LY2334737) and confers prodrug sensitivity to cancer cells. Clin Cancer Res. 2013;19(5):1159-1168.

43. Elsadek B, Kratz F. Impact of albumin on drug delivery - new applications on the horizon. J Control Release. 2012;157(1):4-28.

44. Misak HE, Asmatulu R, Gopu JS, et al. Albumin-based nanocomposite spheres for advanced drug delivery systems. Biotechnol J. 2014;9(1): $163-170$. 


\section{Supplementary material}

\begin{tabular}{|l|l|l|}
\hline Fluorescent intensity & Mean & SD \\
\hline NC & 5.309 & 0.3 \\
\hline Gem & 7.735 & 0.25 \\
\hline Gem-C14 & 10.56 & 0.54 \\
\hline Gem-HSA-NP & 14.035 & 0.65 \\
\hline
\end{tabular}

Fluorescent intensity comparison

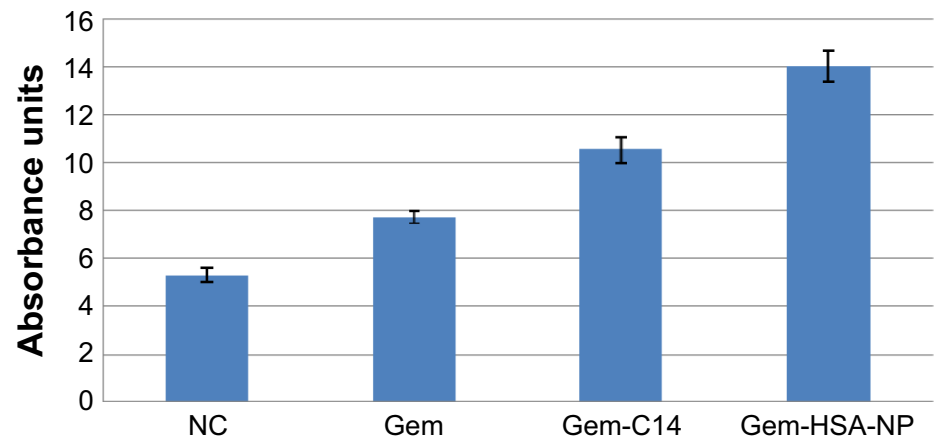

Figure SI Comparison of the average fluorescent intensity in NC, Gem, Gem-Cl4, and Gem-HSA-NP groups.

Notes: Image J software was used to compare the average fluorescent intensity. Data showed a significant difference during the four groups ( $P<0.05)$.

Abbreviations: SD, standard deviation; NC, negative control; Gem, gemcitabine; Gem-CI4, 4-N-myristoyl-gemcitabine; Gem-HSA-NP, gemcitabine-loaded human serum albumin nanoparticle.

\section{Publish your work in this journal}

The International Journal of Nanomedicine is an international, peerreviewed journal focusing on the application of nanotechnology in diagnostics, therapeutics, and drug delivery systems throughout the biomedical field. This journal is indexed on PubMed Central, MedLine, CAS, SciSearch $\AA$, Current Contents $\AA /$ Clinical Medicine,
Journal Citation Reports/Science Edition, EMBase, Scopus and the Elsevier Bibliographic databases. The manuscript management system is completely online and includes a very quick and fair peer-review system, which is all easy to use. Visit http://www.dovepress.com/ testimonials.php to read real quotes from published authors. 